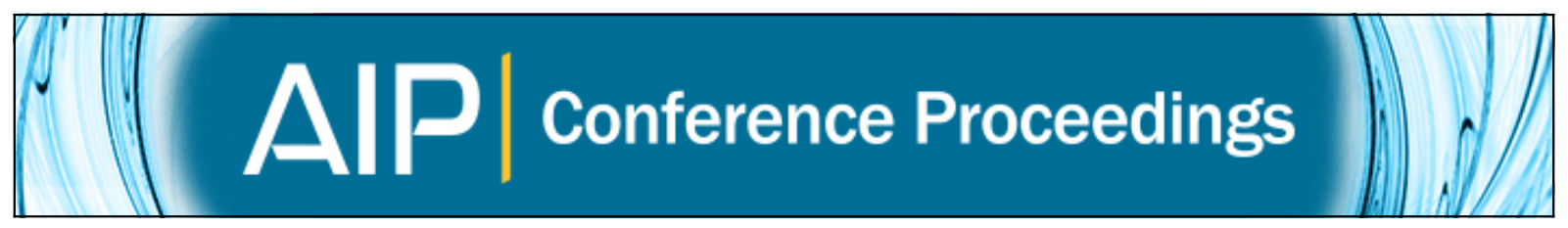

\title{
Preface to the special session Approximation theory and its applications
}

Maria Grazia Russo and Alessandra De Rossi

Citation: AIP Conference Proceedings 1776, 070001 (2016); doi: 10.1063/1.4965347

View online: http://dx.doi.org/10.1063/1.4965347

View Table of Contents: http://scitation.aip.org/content/aip/proceeding/aipcp/1776?ver=pdfcov

Published by the AIP Publishing

Articles you may be interested in

Preface to the special session High performance computing in modeling and simulation

AIP Conf. Proc. 1776, 080001 (2016); 10.1063/1.4965358

Preface to the special session Numerical global optimization algorithms

AIP Conf. Proc. 1776, 060001 (2016); 10.1063/1.4965335

Preface to the special session Intractable problems and approximation algorithms

AIP Conf. Proc. 1776, 050001 (2016); 10.1063/1.4965322

Preface to the special session Numerical and computational methods in data analysis and classification AIP Conf. Proc. 1776, 040001 (2016); 10.1063/1.4965313

Preface of the "Symposium on approximation theory and special functions"

AIP Conf. Proc. 1558, 1119 (2013); 10.1063/1.4825703 


\title{
Preface to the Special Session Approximation Theory and its Applications
}

\author{
Maria Grazia Russo ${ }^{1, a)}$ and Alessandra De Rossi ${ }^{2, b)}$ \\ ${ }^{1}$ Department of Mathematics, Computer Science and Economics, University of Basilicata, Italy \\ ${ }^{2}$ Department of Mathematics, University of Torino, Italy \\ a) mariagrazia.russo@unibas.it \\ b) alessandra.derossi@unito.it
}

The Session has been devoted to some new results in classical topics of Approximation Theory such as polynomial approximation, interpolation, radial basis functions, spline functions, orthogonal polynomials, partition of unity methods. In particular, it has been shown the impact of these new results in the applications such as the numerical methods for integral equations and partial differential equations, the approximation of surfaces, the numerical solution of models in Applied Sciences.

In the following, we briefly describe the contents of the various papers, listed in the order they were presented during the Session.

Paper 1, by Rebelo and Morgado discusses the introduction of graded meshes in the approximation of Caputo derivatives of a function which may be nonsmooth. These graded meshes are constructed through a graded exponent which is determined taking into account the type of singularity of the function allowing to obtain a mesh which is refined near the singular points. Applications to fractional differential problems are given.

Paper 2, by Cavoretto, De Rossi and Perracchione deals with the challenging computational issue of interpolating large data sets, with eventually non-homogeneous densities. To such scope, by minimizing an a priori error estimate, the authors propose a radial basis function partition of unity method by suitably selecting variable shape parameters and subdomain sizes.

Paper 3, by Allasia, Cavoretto and De Rossi considers the problem of interpolating a function given on scattered points using Hermite-Birkhoff formulas on the sphere and other manifolds. For this purpose the authors express each proposed interpolant as a linear combination of basis functions, the combination coefficients being incomplete Taylor expansions of the interpolated function at the interpolation points. The construction of such interpolants takes advantage of not requiring any solution of linear systems.

Paper 4, by Piret concerns the application the RBF-Finite Difference (RBF-FD) technique to the RBF-OGr method for building sparse differentiation matrices discretizing continuous differential operators such as the LaplaceBeltrami operator. The author illustrates the accuracy of the new method by solving the heat equation on the unit sphere.

Paper 5, by Occorsio and Russo proposes a new quadrature scheme of product type based on the so called extended interpolation. Two sequences of product quadrature rules for weakly singular integrals defined on the real semiaxis are constructed, both convergent and stable. The first one uses the classical interpolation based on the zeros of the Laguerre weight and the other the extended interpolation based on two different classes of Laguerre zeros. The mixed sequence allows a strong computational saving.

Paper 6, by Francomano, Hilker, Paliaga and Venturino considers the study of an epidemiological predator-prey model. It analyses the spread of an infectious disease with frequency dependent and vertical transmission within the predator population. Moreover, the authors present a study to analyse the basins of attraction of the stable equilibrium points. A meshless approach has been adopted to produce an approximation of the separatrix manifold.

Paper 7, by Ala, Francomano and Paliaga focuses on the enhancement in accuracy approximating a function and its derivatives via smoothed particle hydrodynamics. Improvements in the solution by reformulating the original method by means of the Taylor series expansion and by projecting with the kernel function and its derivatives, are 
discussed. The improved formulation has been assessed with gridded and scattered data points distribution and the convergence has been analysed referring to a case study in a 2D domain.

Paper 8, by Di Tommaso, Dell'Accio and Hormann, introduces and studies an enhanced triangular Shepard-type operator, based on quadratic Bernoulli polynomials. This operator is a generalization of the Little bivariate Shepardtype operator. Some interesting properties can be deduced for the introduced operators. Approximation error estimates for suitable class of functions are also proved.

Paper 9, by Dell'Accio, Di Tommaso and Hormann deals with the problem of univariate Hermite-Birkhoff interpolation. It is well known that this type of interpolation does not always have a unique polynomial solution. Hence, the authors propose to face an unsolvable problem, first splitting it in two or more uniquely solvable subproblems and then to blend the subproblem solutions together, using multinode rational basis functions as blending tools, in order to get a global interpolant.

Paper 10, by Serafini, gives a strategy in order to compute double integrals defined on the triangle or on the square. The kernels of four variables of the integrals are weakly singular or nearly singular. The idea is to approximate the "regular" part of the integrand function by means of a suitable Lagrange interpolating polynomial in two variables. The convergence and the stability of the procedure is proved.

\section{THE SPECIAL SESSION ORGANIZERS}

Maria Grazia Russo is Associate Professor of Numerical Analysis at the University of Basilicata since 2010. Formerly she was Assistant Professor at the same University since 1995. She received the PhD degree in Applied Mathematics and Computer Science at the University of Naples Federico II (Italy) in 1998. She is actually the Head of the Professors Council of the Computer Science courses of the University of Basilicata. She has published about thirty research articles, counting original articles in international journals and chapter of books. She referees regularly for several international journals in the frame of numerical analysis and applied mathematics. She organized some international conferences and workshops: FAAT 2004 (Maratea, Italy), FAAT 2009 (Maratea, Italy), IWATA 2013 (Rifreddo, Italy), Special Session Special Functions, Orthogonal Polynomials and Application in FJIM 2014 (Bilbao, Spain). She regularly takes active part in international conferences with contributed talks. She held some invited conferences at foreign university. Her main research interests are: polynomial approximation, Lagrange interpolation, polynomial inequalities, quadrature formulas, approximation of integral transforms, numerical methods for integral equations.

Alessandra De Rossi is Assistant Professor of Numerical Analysis at the University of Torino since 1999. She received the $\mathrm{PhD}$ degree in Computational Mathematics at the University of Milano (Italy) in 1997. She has published more than fifty research articles in international journals, chapter of books and conference proceedings. She referees regularly for several international journals in the frame of numerical analysis and applied mathematics. She organized some international congresses, workshop and sessions: DWCAA09 (Canazei, Italy), Special Session Interpolation and Approximation Theory in CMMSE09 (Gijon, Spain), DWCAA12 (Canazei, Italy), MPDE14 (Turin, Italy), CAMO15 (Turin, Italy), Miniworkshop Kernel-based methods and function approximation 2016 (Turin, Italy). She regularly takes active part in international conferences with contributed talks. Her main research interests are: radial basis function method, partition of unity method, Shepard method, fast algorithms for scattered data interpolation, spherical interpolation, image registration, biomathematics. 